\title{
A SURVEY AND REQUIREMENTS OF WEED CONTROL EQUIPMENT FOR NEW ZEALAND
}

\author{
By T. N. GERRARD, Ivon Watkins Ltd., New Plymouth
}

\section{SUMMARY}

WeED control equipment available to the New Zealand user today has passed through many stages of adaption and development since the mid 1940's. The author details this development work and examines various types of equipment at present available. The design of more satisfactory equipment he feels will come about only by closer liaison between chemical research work and the agricultural research engineer.

As this conference is devoting its whole time to chemical weed control, it is unnecessary to discuss the mechanical tillage implements which play an important part in another branch of weed control. Therefore my remarks will be confined to equipment suitable and otherwise for applying weed control chemicals generally.

To appreciate the changes in spray equipment suitable for farm and contractor use it is important to go back 10 years or. so and review the early types. Undoubtedly, the first equipment used was the knapsack type which even today is unaltered. The greatest change, however, has been in the so called power sprayers. Whereas first a compressed air liquid combination was used it was not long before these were found to be bulky, expensive, and dangerous.

A rapid change to the complete liquid reticulation system was a welcome and practical move for simple and efficient operations to be carried out by all types of users..From such simple beginnings, however, we were soon to see a very disorganised and somewhat negative development of spray equipment, principally due to the following factors:

Chemical weed control was the result of work by many people outside the true agricultural field and who gave little thought to farm operations.

The agricultural engineer was either sceptical of this new method or was not co-opted to develop application equipment as a project along with the chemicals.

The new chemicals were in many cases being manufactured and distributed by organisations who were new in the agricultural field and were without engineering staff, as I am sure it was not envisaged that these new chemicals and engineering would have to go hand in hand.

To overcome this obstacle of no application equipment the obvious course of adaptation was resorted to in that standard engineering items were adapted and a temporary type of equipment evolved. At this early stage it was hoped that manufacturing engineers would provide in the future suitable equipment, but as we know today the bulk of weed control equipment is - a direct result of work by the organisations devoted to weed control.

The result of early adaptations and make-do was no doubt responsible for the variety of spray equipment available to the user, and rather than benefit him it often confused the issue. At this stage qualified workers were being hired in the chemical branches of weed control, but the development of equipment was a matter of commercial expedient rather than development by qualified engineers. The employment of engineers is most essential is we are to have sound development and trials. 


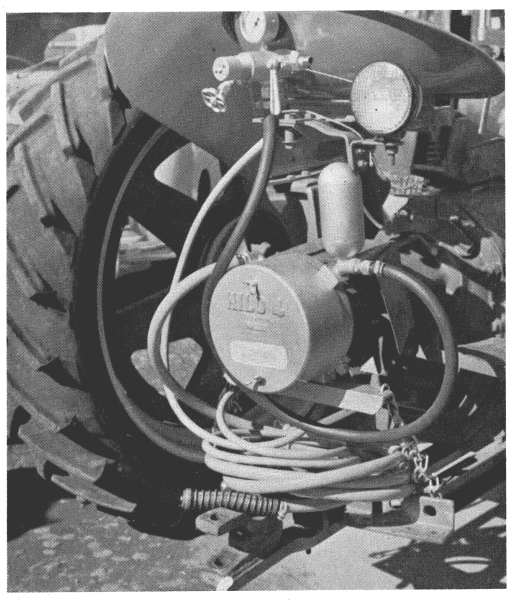

A p.to. operated high and low pressure plunger pump. 


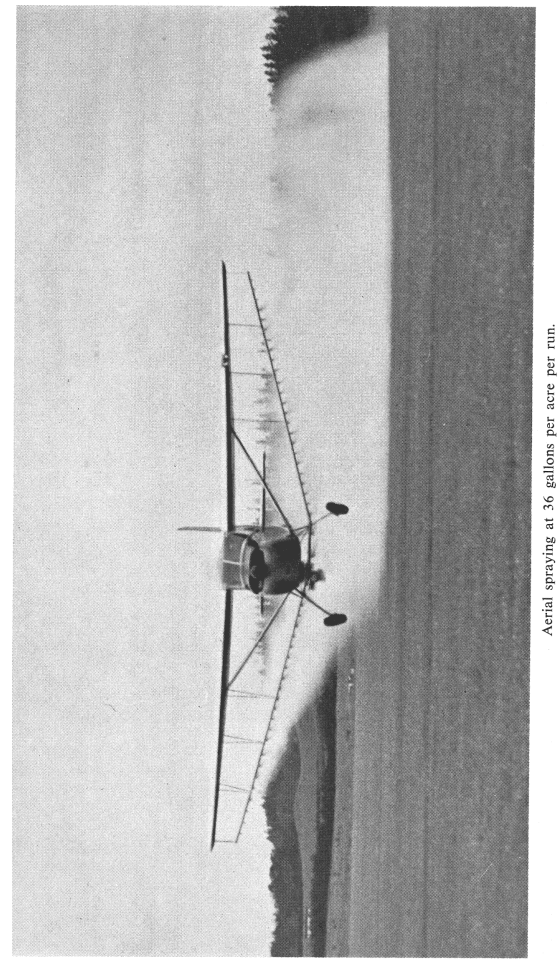




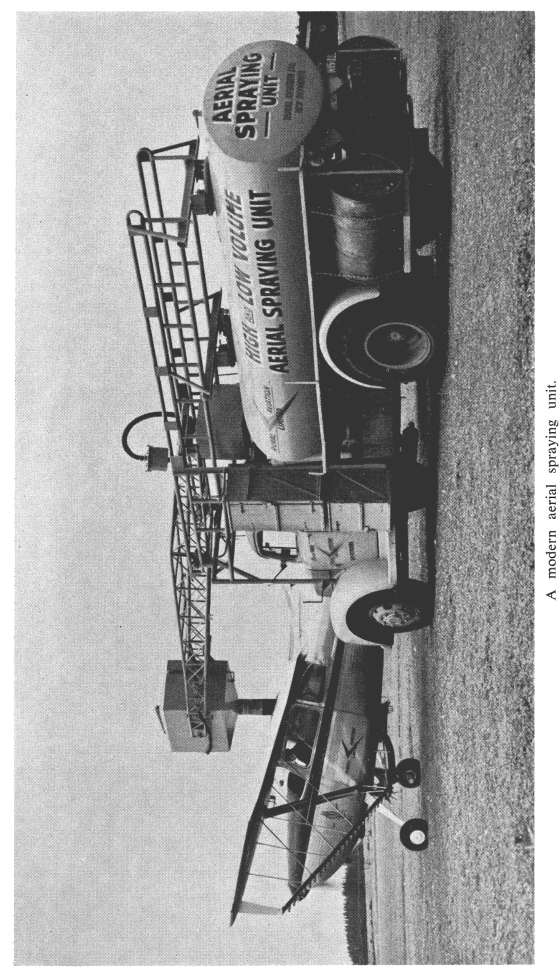




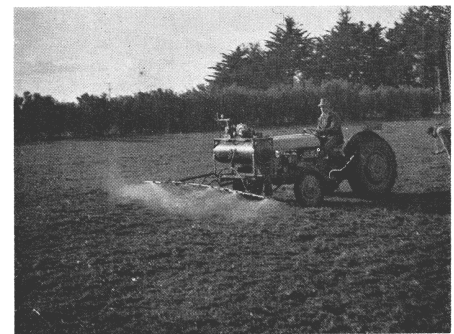

An air-liquid low volume sprayer. 
The introduction of 2,4,5-T for brush control further complicated the matter of standardisation on spray equipment and once again the user was faced with a variety of types of equipment. As weed control at this time had shown its potential use in agriculture, it is interesting that no effort had been made to co-ordinate chemical trials and equipment trials so as to present full and complete data for presentation to the user.

Little other work on equipment other than that carried out by $\mathrm{Mr}$ Crosbie on nozzle patterns has been presented to this conference and it could well be from today on that some co-ordinated work will be carried out for the benefit of weed control in general.

To enable us to discuss the requirements of weed control equipment it is first necessary to section the users of weed control according to their activities. Our first division is between farmer and non-farmer, the lowland farmer and the hill country farmer. Our non-farmer can be broadly divided between contractor and domestic. These could be further subdivided, but as the bulk of weed control is applicable to farming operations either by the farmer himself or by contract, these will suffice to give a fair crosssection of equipment requirements.

The lowland farmer should be the easiest to provide for in general, but unfortunately the equipment suitable for high pressure, low pressure, high volume, low volume is not always available at a price to suit his pockef. This is in some cases brought about by one chemical such as a wettable powder or suspension in mixture with a soluble material making a rotary type pump unsuitable. This type of pump is undoubtedly the least costly and fulfils the general requirements of the low country farmer.

The hill country farmer up to the present has not been a user of pasture spraying equipment, but the advent of the chemical method of pasture renovation has opened a new field of equipment usage. I would like to see suitable equipment trials carried out by the Department of Agriculture in conjunction with chemical trials, as I believe various oversowing and undersowing implements have been tested in these trials.

The hill country farmer who has practised gorse control has always been faced with much heavier expenditure for high pressure work than the low country farmer. In these cases when heavy pumping for long hours at high pressures is necessary the plunger pump has remained in firm favour.

The hill country farmer, too, has been the inspiration of aerial spraying in New Zealand, particularly for thistle and lately gorse control.

This problem of satisfactory equipment for high volume aerial application has not been given the attention due to it except by a few individuals and aerial operators who realise the potential of such equipment. Before entirely satisfactory equipment is available to apply rates of 40 to 50 gallons per acre per run much further development work will need to be done.

The introduction of the helicopter for aerial spraying operations offered new hope to the hill country farmer and while the results to date may have been variable in gorse control, the chemical method of pasture renovation of hill country land could well be the prerogative of the helicopter. Again full trials will be required to establish data applicable to this agricultural operation.

The weed control contractor is faced with a wide variety of problems in selection of his equipment to cope with a wide variety of jobs, but the basic items which he is most concerned with are pumps, engines, hoses, booms, and spray guns.

The items giving greatest trouble are hoses and booms. Only the purchase of first-quality hose can be warranted, but boom design is of a different character and I would recommend that for contractor use a cantilever type be constructed for each individual case. 
A broad outline having been given of the main users of weed control equipment and the basic requirements of each case, a more detailed examination of types and parts of weed control equipment is given in the following paragraphs:

There is a definite tendency to avoid the use of small engine powered spray units and concentrate on the p.t.o. operations. The most favourable argument is why have another engine on the farm?-and is fully justifiable under many conditions provided suitable equipment is designed for p.t.o. operation by tractor, etc. As the h.p. required seldom exceeds 3 to 4 h.p. at 400 to 500 r.p.m., the farmer particularly does not like running his tractor of 30 to 50 h.p. at full operating revs. when the tractor is stationary.

However, under normal pasture spraying conditions full p.t.o. speeds are realised and light loading of the engine is avoided.

The various non-positive displacement rotary pumps and gear pumps available fulfil the general requirement for p.t.o. operation at normal operating speeds with the tractor in motion. These pumps give good volume at medium pressures and give varying degrees of life depending on the quality.

It is the p.t.o. operated high pressure pump which is also required to do low pressure spraying which has given the engineering people the most trouble. For static work p.t.o. speeds of 250 to 300 r.p.m. are generally accepted by the farmer and engineer alike as not being unnecessarily severe on the tractor engine. The accomplishment of pressures of 250 to 400 per square inch and 400 gallons per hour consistent with a compact pump adaptable for p.t.o. operation has been the subject of many ingenious schemes both by plunger and rotary pumps. Positive displacement plunger pumps have a wide range of operating speeds and pressures and are consequently more adaptable and versatile; they will also handle wettable powders, etc.

The rotary pump of centrifugal, vane, or roller design requires a higher r.p.m. than is warranted under static conditions.

Unfortunately, up to the present no successful positive or non-positive displacement rotary pump has been designed which meets the wide range of duties required under New Zealand conditions. This could be a line of research on the application equipment which I am sure would be well warranted.

It is pleasing to note that most suppliers are fitting efficient damping devices to cancel out fluctuation of pressure gauges to enable more accurate readings and setting to be made. Too often misapplication has been the result of faulty pressure gauges.

A very important part of any spray equipment is the pressure regulator and here again we have a wide variation in design and adaptations. A pressure regulator must fulfil two functions: 1. Pressure regulation. 2. Avoid excessive pressure build up during "off" cycles.

The majority of low pressure regulators available have been a carry-over from the early days of small low capacity pumps and the time is now past when such inefficient units are accepted as satisfactory.

The fitting of a single movement control valve should become general practice to avoid unnecessary over-spraying and uneven commencement of spray runs during pasture spraying.

The problem of supplying a satisfactory hose for general use has been complicated by spraying at high and low pressures.

Fortunately, our climatic conditions have allowed the use of plastic hoses in low pressure units, but in the case of the dual purpose unit only 
imported reinforced hose has met the requirements. A common fault of hoses suitable for 250 p.s.i. working pressures has been the inadequate protective outer rubber cover which is easily damaged or penetrated by thorns, etc., resulting in premature damage to the reinforcing fabric.

The types of spray guns available are an indication of an increasing awareness by manufacturers that considerable waste of material has taken place when the semi-rotary shut-off was generally accepted. The introduction of trigger type guns is to be commended for efficient and practical use.

In pasture spraying by boom methods, though the one piece boom is satisfactory for the smaller user, little development work has gone into the design of a satisfactory articulated type for our New Zealand conditions. However, I believe manufacturers are looking closely at this problem.

Probably one of the most important and yet smallest parts yet to be mentioned in detail is the spray nozzle. There is a wealth of good data available on spray nozzles of all kinds relating to specific design, droplet range, and spray pattern.

The range of spray nozzles on the New Zealand market fortunately is limited to a few well tried and reliable makes. However, within some manufacturers' range there is a wide choice of agricultural spray nozzles which I believe rather complicates the issue of type selection for New Zealand.

As our application rates particularly for pasture weed control are generally higher than that of, say, Australia and U.S.A. the simple fan nozzle gives adequate coverage and does increase the material selectively; sufficiently to recommend its use where clovers are important

For the hard-to-kill weeds such as ragwort and the application of contact sprays the solid cone nozzle should find more favour than at present.

I shall be most interested to hear a later paper on nozzle design and selection.

When discussing the use of pressures in excess of 250 p.s.i. for average farmer use with many people I am unable to produce sufficient evidence to prove this is necessary. I will qualify this statement, as in instances reported of poor brush control that I have examined, the use of pressures in excess of 250 p.s.i. in inexperienced hands has resulted in indifferent coverage and retention due to excessive fogging of the spray material. I believe this high pressure spraying has been a carry-over from adaptation of orchard spraying equipment and I am sure this matter of high pressure spraying warrants full and complete investigation to further our knowledge of weed control generally.

If weed control spraying had continued to follow orchard spraying, much lower pressures would be used today. I think this high pressure spraying is a good example of adaptation for the moment with no follow up or research.

\section{CONCLUSIONS}

Briefly then we require for New Zealand weed control:

Equipment without auxiliary engines, except in special circumstances, which is adaptable for p.t.o. operation at 250 to 300 r.p.m. and outputs of up to 400 gallons per hour to cover both high and low pressure ranges.

An efficient and reliable pressure regulator unit with dampened pressure gauge, solvent resistant hoses applicable to the pressure involved, and either spray boom with known pattern nozzles or a quick shut off spray gun. This equipment must be rugged, reliable, and easily maintenanced and adaptable to a wide range of users' intelligence. 
Variations and additions to the basic requirements are to be commended for efficient and practical mounting to tractors, trucks, etc.

Having given I trust a fair outline of the history and development of weed control equipment in New Zealand I suggest that some considerable effort be made by those interested parties at this conference to encourage and develop complete liaison between chemical research workers and agricultural engineers in an endeavour to improve weed control techniques and equipment more suitable for New Zealand conditions.

No matter how good a particular weed control chemical may be, unless it is capable of being applied easily, accurately, and effectively under field conditions with the range of equipment available, much valuable work may be lost. Therefore, I believe due importance should be attached to parallel development in field trials of chemicals and equipment to complete recommendations of application rates and method.

\section{DISCUSSION}

Q.-What type of pump is recommended for use with insoluble wettable powders, e.g. monuron?

A.-The plunger type is the most suitable for abrasive materials.

Q.-Are manufacturers investigating the fitting of lining to plastic hose to improve its durability?

A.-Braided plastic hose was tried, but was costly and fairly heavy, and as the braid was on the outside, it did not slide easily. Plastic Hose with internal braiding presents manufacturing problems.

Q.-As pressure gauges on high-pressure units are only occasionally referred to during spraying, could this life not be increased by providing a cock to close the gauge off when not required?

A.-Such cocks were used until a sufficiently rugged gauge was developed.

Q.-Could not weight-loaded valves such as used on the Ruakura milking machine be used on spraying units in place of pressure gauges?

A.-Such valves should be used in a vertical position and are suitable for use on stationary devices only, such as a milking machine. 